\title{
Las emociones de niños y adolescentes frente a la educación virtual debido a la crisis sanitaria
}

DOI: https://doi.org/10.33262/ap.v3i4.1.126

\begin{abstract}
(c) (1) (5) (2)
The emotions of children and adolescents in the face of virtual education due to the health crisis
\end{abstract}

Ángela Rosario Flores Vélez. ${ }^{1}$, Leticia Cedeño Macías. ${ }^{2}$, Rosalyn Cedeño Zambrano. ${ }^{3}$ \& Ángela Katherine Vera Moreira. ${ }^{4}$

\begin{abstract}
Introduction. With the appearance of the sanitary emergency provoked by the COVID 19 , the education field turned completely virtual, it was in order to solve the quarantine situation which didn't allow students to go to schools, colleges and universities. With this new educative system, which all people was forced to, new challenges appeared due to the fast changes. No students or teachers were well prepared for this alterations. Because of this situation, a question appears: which are the children emotions facing the virtual education during the pandemic time? Objective. In order to answer this question, this paper propose the objective of determine the emotions children feel facing the virtual education during the pandemic time. Methodology. In order to get the results, this paper was based in a qualitative and ethnographic investigation, which searched to do a detailed description related to children emotions facing the virtual classes during the pandemic. Results. These results showed that children had positive emotions and feelings like surprise, happiness and enthusiasm, but they also experienced fear and frustration, most

\footnotetext{
${ }^{1}$ Docente Universidad Técnica de Manabí, Facultad de Filosofía, Letras y Ciencias de la Educación, Carrera de Pedagogía de los Idiomas Nacionales y Extranjeros angela.flores@utm.edu.ec ORCID iD http://orcid.org/0000-0002-5495-7815

2 Docente Universidad Técnica de Manabí, Facultad de Filosofía, Letras y Ciencias de la Educación, Carrera de Pedagogía de los Idiomas Nacionales y Extranjeros leticia.cedeno@utm.edu.ec ORCID iD http://orcid.org/0000-0002-7263-3902

3 Docente Universidad Técnica de Manabí, Facultad de Filosofía, Letras y Ciencias de la Educación, Carrera de Pedagogía de los Idiomas Nacionales y Extranjeros, rosalyn.cedeno@utm.edu.ec https://orcid.org/0000-0002-7683-5317

${ }^{4}$ Docente Universidad Técnica de Manabí, Facultad de Filosofía, Letras y Ciencias de la Educación, Carrera de Pedagogía de los Idiomas Nacionales y Extranjeros, angela.vera@utm.edu.ec ORCID iD 0000 0002-2540-340X https://orcid.org/my-orcid?orcid=0000-0002-2540-340X
} 
of the time for not having the technological issues needed to attend their classes. Conclusion. This paper conclude that children and teenagers are facing positive and negative emotions, however, most of them feel fear and frustration due to a educative system they do not understand, because they don't feel prepared to deal with it due to their lack of knowledge and technological devices.

Keywords: emotions, virtual education, pandemic, adolescent, children.

\section{Resumen}

Introducción. Ante la emergencia sanitaria provocada por el COVID 19, el sector educativo se tornó completamente virtual, con el objetivo de dar solución a la cuarentena que impedía que los alumnos asistieran a las aulas. Con este nuevo sistema educativo, impuesto a la fuerza de la necesidad, surgieron nuevos desafíos provocados por el rápido cambio, para el que ni docentes ni estudiantes estaban preparados. Ante esta situación, surge la problemática de conocer ¿Cuáles son las emociones de los niños y adolescentes frente a la educación virtual debido a la crisis sanitaria provocada por la pandemia del covid-19? Objetivo. Determinar las emociones que experimentan los niños y adolescentes frente a la educación virtual debido a la crisis sanitaria provocada por esta pandemia. Metodología. este trabajo se basa en una investigación de enfoque cualitativo de tipo etnográfico, en virtud de que buscó describir la situación actual de los niños y adolescentes en relación a las emociones que experimentaban frente a la educación virtual por la crisis sanitaria. Resultados. Los resultados mostraron que los niños, niñas y adolescentes evidencian sentimientos y emociones como la sorpresa, alegría y entusiasmo, pero también se hallaron sentimientos de miedo y frustración, que los niños experimentan ante el hecho de no poseer el servicio de internet y dispositivos tecnológicos, Conclusión. Se concluyó que los niños y adolescentes enfrentan diferentes emociones, tanto positivas como negativas, sin embargo, la mayoría de ellos sienten miedro y frustración por un sistema de estudios que no entienden, para el que no están preparados ni cuentan con las herramientas necesarias.

Palabras clave: emociones, educación virtual, pandemia, niños, adolescentes.

\section{Introducción}

La educación virtual o enseñanza en línea describe el proceso de enseñanza-aprendizaje desarrollado con la utilización de medios tecnológicos, conocidos como TICs o Tecnologías de la Información y la Comunicación. En este entorno, la interacción entre los actores educativos, se lleva a cabo en coincidencia de tiempo, sin coincidir en espacio; o sin coincidir en tiempo y espacio (Duart \& Sangrà, 2000). 
Actualmente se distinguen cuatro tipos de aprendizajes electrónicos: eLearning, bLearning, mLearning y ULearning (Moll, 2019). Estos tipos de aprendizaje permiten dirigir el proceso de enseñanza de tal forma que el aprendizaje sea significativo, mientras se desarrollan capacidades autónomas; el estudiante es capaz de asumir un rol activo a través del dominio y establecimiento de las actividades pertinentes para su formación como fijarse objetivos o momentos, evaluarse, entre otros.

La actividad educativa que utiliza el internet y los medios electrónicos, se la define como eLearning, mientras que la educación que fusiona la presencialidad y no presencialidad se la denomina bLearning. Por otro lado, mLearning se caracteriza por el uso de dispositivos móviles tanto dentro como fuera del aula, mientras que uLearning es el tipo de aprendizaje ubicuo y omnipresente donde la comunicación se da en tiempo real (sincrónica) o sin converger en un mismo momento (asincrónicas).

Debido a la emergencia sanitaria que el mundo ha vivido desde la aparición del COVID19, la educación virtual ha sido el único medio en que los procesos de enseñanza aprendizaje de todos los niveles educativos ha podido desarrollarse. La UNESCO, a través de múltiples programas ha apoyado los procesos educativos a través de varios proyectos que hacen un análisis y proponen alternativas en campos como el rol docente, el impacto curricular y las evaluaciones (UNESCO, 2020). Esto mismo proyecto, también se preocupa de la salud mental de los estudiantes en América Latina, para lo cual propone algunas estrategias que buscar favorecer la motivación de los alumnos hacia los estudios online.

El Ministerio de Educación del Ecuador (2020), en respuesta a la suspensión de clases por la emergencia sanitaria y con el fin de garantizar la continuidad del servicio educativo propone inicialmente el plan "Aprendemos juntos en casa", en este se sugieren los recursos digitales o virtuales como medios de comunicación, y se resaltan los medios de comunicación como la televisión y la radio, en otras palabras, se favorece la teleeducación.

Para el proceso de formación de los estudiantes en casa, se tienen a disposición 160 canales de televisión con el programa educativo "aprender la Tele"; 1000 radios ruralescomunitarias en 3 franjas de programas educativos; acompañamiento docente; fichas pedagógicas; portal educativo: recursos2.educación.gob.ec y plataforma para estudiantes de tercero de Bachillerato: ava-bachillerato.educación.gob.ec. (Ministerio de Educación del Ecuador, 2021).

Además de los recursos mencionados anteriormente, el trabajo en casa o autónomo ha sido una característica en la continuidad de la educación. En este contexto la planificación de actividades flexibles para ser desarrollada por niños y jóvenes, así como también el rol de acompañamiento por parte del docente son aspectos propios de este proceso.

En este escenario, las emociones de quienes conforman la comunidad educativa han tenido un rol muy significativo. Emociones que resultan en afectaciones de la salud mental (Johnson et al., 2020). Entre las principales afectaciones que se pudieran derivar 

noviembre, 2021

en la fase inicial del confinamiento están el miedo, la incertidumbre y la angustia. Por otro lado, están aquellas derivadas del cambio de actividad como el desorden o trastorno del sueño (Gálvez, 2021), que de cierto modo están interrelacionadas.

Se suman a estas emociones las derivadas por el acceso de medios tecnológicos por parte de docentes y discentes. En el campo Sánchez et al. (2020) ha resaltado la importancia de la docencia, donde: "la formación pedagógica, disponibilidad de recursos digitales, recursos informativos sobre uso de tecnologías en educación, mejora de procesos institucionales, acompañamiento pedagógico, asesoría técnica e información de los recursos institucionales" (p.1). Así mismo la brecha tecnológica, procedente de las desigualdades sociales de la población discente, impiden el acceso o continuidad en la educación, generando emociones propias de la desigualdad (Anderete, 2020), redundando en las mencionadas anteriormente.

Las emociones según la Asociación Española contra el Cáncer (2021) son: "mecanismos que nos ayudan a reaccionar con rapidez ante acontecimientos inesperados que funcionan de manera automática, son impulsos para actuar" (p.4), es decir que son el resultado de la experiencia subjetiva, la reacción fisiológica y la respuesta conductual. Las emociones básicas son miedo, asco, enfado, sorpresa, felicidad y tristeza (Garrido, s.f.), es decir que las emociones pueden ser tanto positivas como negativas.

No obstante, pese a esta clasificación Bueno y Torrens (2019) señala que existen al menos cuarenta emociones que se derivan de las señaladas por Garrido y que son:

“el aburrimiento, la aceptación, el afecto, el agradecimiento, la agresión, la ambivalencia, el amor, la ansiedad, la apatía, la compasión, la confusión, la culpa, la depresión, el desprecio, el dolor, la duda, la empatía, la envidia, la esperanza, la euforia, el éxtasis, la frustración, el hambre, la histeria, el horror, la hostilidad, el interés, el lamento, la lástima, la nostalgia, el odio, el orgullo, la paranoia, el perdón, el placer, la rabia, el remordimiento, la simpatía, la soledad, el sufrimiento y la vergüenza"

Según Bueno y Torrens (2019) las emociones son "patrones de comportamiento que se desencadenan ante el cambio en el statu quo", situación a la que se vieron expuestos los estudiantes por el cambio que se llevó a cabo en sus vidas y particularmente en cómo se desarrollaban sus clases. Las encargadas de generar las emociones en nuestro cerebro son las amígdalas y en cada hemisferio cerebral hay una, es decir, una en el hemisferio derecho y otra en el izquierdo.

Según Bueno y Torrens (2019), estos comportamientos o emociones luego se racionalizan y se convierten en sentimientos que pueden ser expresados a través de las palabras. Según este autor la relación que tienen las emociones con el aprendizaje se basa en que cuando éste se genera ante alguna acción, el cerebro lo almacena y lo utilizará de forma más eficiente. 

noviembre, 2021

Al respecto Fernández (2017) señala que cuando existe la sobreestimulación del cerebro, es decir, el estudiante es sometido a presión, ya sea por el esfuerzo del mismo o por la dificultad en realizarlo, las neuronas segregan una sustancia llamada cortisol, que es el neurotransmisor que produce el estrés, mismo que en elevados niveles. Según Bueno y Torrens (2019) constituye en un bloqueo para el aprendizaje, por lo tanto los docentes deben evitar en lo posible someter a los estudiantes a actividades que los estresen excesivamente, porque a decir de este autor, un estrés moderado, es inclusive conveniente para despertar en el estudiante el deseo de lograr el desafío; por lo tanto se recomienda la planificación de tareas y actividades tomando en consideración las inteligencias múltiples que poseen los estudiantes, su edad y preferencias y otros aspectos que potencialicen la motivación y la sorpresa.

La educación se caracteriza por promover espacios de interacción entre el maestro y los estudiantes, que generan el aprendizaje. Esta sensación de acercamiento da como resultado emociones positivas que traen consigo la posibilidad de alcanzar los objetivos trazados en el proceso de enseñanza-aprendizaje (García, 2012). Los encuentros educativos mediados por entornos virtuales pueden desatar emociones positivas (Lurich et al., 2015), sin embargo, por las necesidades educativas expuestas anteriormente, aún se pueden plantear acciones de mejora.

Otro factor a considerar, está relacionado con las emociones y la capacidad de adaptación a los entornos virtuales. Para lograr este proceso de adaptación y generar emociones positivas, es necesario contar con una metodología instruccional, interfaz de usuario, organización de contenido, nivel de interactividad del entorno, requerimiento de participación, trabajo colaborativo, prácticas evaluativas, y sistema tutorial, todo esto atendiendo a los diversos estilos de aprendizaje como el afectivo, reflexivo, pragmático y teórico (Del Moral \& Villalustre, 2005).

\section{Metodología}

La presente investigación se llevó a cabo como producto de un trabajo intra aula con los estudiantes del II semestre de la Carrera de Pedagogía de los Idiomas y Lingüística de la Universidad Técnica de Manabí, establecido en el respectivo plan de clases, para cumplir el resultado de aprendizaje, el mismo que buscaba utilizar los principales métodos, técnicas e instrumentos de la investigación cualitativa evaluando criterios de validez y confiabilidad para la aplicación de los diferentes procedimientos.

Se basa en una investigación de enfoque cualitativo de tipo etnográfico, en virtud de que buscó describir la situación actual de los niños y adolescentes en relación a las emociones que experimentaban frente a la educación virtual por la crisis sanitaria.

La población considerada fueron los estudiantes de las unidades educativas de los niveles básico y bachillerato del cantón Portoviejo, que reciben clases virtuales debido a la crisis sanitaria por la COVID 19. 

noviembre, 2021

La muestra se basó en un método no probabilístico circunstancial, debido a que la situación que se atravesaba solo permitió acceder a los participantes que estaban disponibles y no se basó en una norma pre establecida (Barrientos, 2013), por lo cual estuvo constituida por 220 estudiantes de las unidades educativas de los niveles básico y bachillerato.

Se utilizó la entrevista a profundidad, que se desarrolló a través de un cuestionario de diez preguntas abiertas, que apuntaban a conocer y analizar las emociones que experimentaban los estudiantes ante las clases virtuales por la emergencia sanitaria, misma que se aplicó a través de llamadas telefónicas y mensajes de WhatsApp, cómo único medio de comunicación entre los investigadores y los investigadores quienes mostraron su predisposición en colaborar con el objetivo trazado.

\section{Discusión y Resultados}

La crisis sanitaria provocada por la pandemia de la COVID 19, ha ocasionado grandes cambios en la sociedad en varios aspectos. Tal es así, que los sistemas económicos, de transporte, sanitarios y de educación en el mundo, se han visto en la necesidad de transformarse con el objetivo de enfrentar las consecuencias de esta enfermedad. Ecuador no está exento de estos cambios, y se ha visto en la obligación de sumarse a ellos por el bienestar de su población.

Alteraciones tan bruscas e inesperadas, generan consecuencias en la psique de las personas, mucho más, cuando se trata de niños y adolescentes, quienes aún no han desarrollado por completo su inteligencia emocional, y cuya inteligencia intrapersonal está lejos de haber alcanzado la madurez necesaria para enfrentar metamorfosis de este grado.

La educación ecuatoriana también sufrió un cambio radical, teniendo que adaptarse a la nueva realidad. Se iniciaron las clases virtuales obligatorias en todas las instituciones de educación del país, sin embargo, las clases en línea, evidenciaron el hecho de que no todas las familias ecuatorianas tenían acceso a internet desde sus casas, ni los dispositivos tecnológicos necesarios para el acceso a la red, lo que provocó en los niños, niñas y adolescentes diversos tipos de emociones que impactan en su proceso de aprendizaje.

Al inquirir a los alumnos entrevistados, sobre lo que más les gusta de las clases en línea, el $95 \%$ de ellos contestó que poder conocer al fin a su profesor (a) y ver a sus amigos e interactuar con ellos. Estos datos se basan en el hecho de que los primeros meses de clases, no se debían conectar al aula virtual, sino que se les enviaban las actividades planificadas a realizar a través del correo electrónico o de redes sociales como WhatsApp o Facebook, que son mucho más accesibles desde cualquier teléfono inteligente, y sin necesidad de computadora. Este dato evidencia la necesidad de los niños, niñas y adolescentes de la convivencia con sus pares y de la compañía, asesoría y guía del profesor.

Los estudiantes concuerdan en que la mayor dificultad que enfrentan en sus clases virtuales es el desarrollo de las tareas, pues, aunque durante los encuentros sincrónicos 

noviembre, 2021

los profesores explican los temas en los cuales se basan las tareas, no siempre quedan claros, y se les dificulta elaborar las tareas sin la supervisión y guía del profesor

El 100\% de los entrevistados exponen el hecho de haber tenido dificultades de diversa índole al incursionar en la educación virtual, sobre todo por el hecho de que este tipo de entorno era desconocido para ellos.

Los estudiantes concuerdan en que la mayor dificultad que enfrentan en sus clases virtuales es el desarrollo de las tareas o actividades prácticas, las cuales antes eran desarrolladas durante las clases bajo la supervisión y guía del maestro, sin embargo, en la realidad actual, en los encuentros sincrónicos los profesores explican los temas en los cuales se basan las tareas, pero carecen del tiempo o los medios para desarrollar actividades prácticas sincrónicas. Este hecho, causa en los alumnos impotencia, estrés y decepción, pues sienten que no aprendieron en la clase sincrónica, que no son capaces de hacer la tarea y se presionan con el hecho de que éstas deben ser presentadas a tiempo y calificadas.

Otra dificultad en que acuerdan los entrevistados es el acceso a internet, el $80 \%$ de ellos no cuenta con el servicio en sus hogares, y sus padres deben invertir en planes con internet en sus teléfonos o buscar diversas alternativas para que ellos puedan atender a sus clases virtuales sincrónicas.

Carecer de dispositivos electrónicos para atender a sus actividades y desarrollar sus tareas es otra de las dificultades que afrontan los entrevistados. Solo uno de cada cinco alumnos tiene una computadora, el 95\% de ellos utilizan sus teléfonos celulares o los de sus padres, lo cual dificulta mucho la elaboración de tareas, de hecho, las hacen de manera escrita y envían fotos de las mismas al maestro para que las califique.

Adaptarse a los entornos virtuales es considerado una dificultad para los entrevistados, quienes nunca, antes de la obligatoriedad provocada por la pandemia, habían tenido experiencia en aprendizaje virtual.

El 30\% de los entrevistados acuerdan en que es muy difícil entender al maestro en los entornos virtuales, tal vez por su inexperiencia en estos entornos, y se les dificulta también el uso de la plataforma virtual de aprendizaje TEAMS, la cual es considerada ineficiente y de difícil acceso.

Al inquirir a los entrevistados qué les desagrada de las clases virtuales, y cómo se sentían con respecto a estos aspectos, los alumnos expusieron que les desagrada no hacer prácticas guiadas por el profesor, la falta de interacción que hay entre ellos y sus compañeros, y el depender de la conexión de internet para sus clases. Todo esto provoca en ellos sentimientos de estrés y frustración. Además, admiten que sienten un poco de miedo, pues las clases virtuales les recuerdan que estamos atravesando una emergencia sanitaria que podría provocarles la muerte a ellos o a cualquiera de sus familiares y amigos. 

noviembre, 2021

Todos están de acuerdo en que las clases virtuales son una buena opción en tiempos de pandemia, porque los mantiene más seguros que asistiendo a clases presenciales, sin embargo, se sienten muy estresados en los entornos virtuales, debido a las dificultades ya mencionadas: fallas o carencia de internet en sus casas, carencia de equipos tecnológicos, etc.

Por último, al indagar las emociones que sienten debido al cambio en la modalidad de estudios provocado por la emergencia sanitaria, expresan una gama variada de respuestas por diversas causas: Alegría y entusiasmo al ver a sus amigos a través de la cámara. Aburrimiento, fatiga y decepción durante los encuentros virtuales por no entender las clases, largas y aburridas, según ellos, y en las que no participan activamente, sino que solo escuchan al profesor. Enojo, estrés y frustración al no poder desarrollar las tareas. Confusión al vivir tantos cambios para los que no se sienten preparados.

A través de las entrevistas realizadas, se concluye que, las niñas, niños y adolescentes sienten distintas emociones al enfrentarse a las clases virtuales en tiempos de pandemia. Ante todo, están conscientes de la necesidad de este tipo de clases durante la emergencia sanitaria para que ellos, sus padres y familiares estén seguros y sanos, sin peligro de contagio de la covid-19. Están bien informados del peligro que significa el reintegro a clases presenciales, por lo tanto, ven las clases virtuales como la mejor opción para continuar con su educación, a pesar que ello les significa dificultades que les hacen experimentar emociones como la confusión, estrés, decepción, frustración, aburrimiento y fatiga. Por otro lado, ansiosos de la interacción con sus compañeros de clases, y la interrelación social con sus amigos, ven en las clases virtuales la oportunidad de ver las caras extrañadas y conocidas, lo cual les hace experimentar emociones de alegría y entusiasmo.

Queda claro que, los eventos relacionados con los procesos de enseñanza aprendizaje que se desarrollan a través de las clases virtuales, provocan emociones negativas en los niños, niñas y adolescentes, y que, el ámbito social-afectivo relacionado con sus compañeros en los entornos virtuales de aprendizaje, provoca en ellos emociones positivas.

\section{Conclusiones}

- Al analizar cada uno de los resultados que se presentan en esta investigación se evidencian sentimientos y emociones como la sorpresa, alegría y entusiasmo, mismos que generan en los cerebros de niños y jóvenes un neurotransmisor llamado serotonina que estimula al cerebro al aprendizaje y por ende permite que los aprendizajes sean significativos. en la relación entre la sorpresa y el aprendizaje la alegría es un sentimiento que transmite confianza.

- En relación a los sentimientos como miedo y frustración, que los niños experimentan ante el hecho de no poseer el servicio de internet y dispositivos tecnológicos, indica que los aprendizajes no son significativos, no se concretas ni complementan con el aprendizaje anterior. Este tipo de sentimientos pueden 
generar rechazo, huida, bloqueo y baja autoestima en los estudiantes, creando una barrera que evita que el aprendizaje se lleve a cabo eficientemente.

- De igual manera uno de los aspectos que causan emociones y sentimientos negativos en la realización de tareas complejas, es el hecho de que éstas son muy difíciles, a decir de los estudiantes, por lo tanto, es importante conocer que un cerebro sobre estimulado produce estrés y éste es el enemigo número uno del órgano rector del cuerpo, ya que el estudiante puede llegar a sentir que al no terminar una tarea puede perder su materia u obtener una mala nota.

- Las situaciones externas que genera el ambiente influyen en el aprendizaje, en la planificación, el razonamiento y la toma de decisiones, debido a que el cerebro como es el órgano de la supervivencia, al sentir una amenaza no realizará conexiones neuronales que son propicias para las habilidades antes indicadas, y por el contrario la amígdala cerebral, que es la memoria emocional, segregará una glándula que generará el estrés, dando como resultado el miedo o la irá como demostración de rechazo a la situación que enfrenta.

- Finalmente, se concluye que la educación virtual, aun siendo la única manera de poder continuar con los procesos educativos, ha generado en la mayoría de los estudiantes miedo, frustración y desmotivación, sobre todo en aquellos estudiantes que no cuentan con el apoyo necesario en casa para la dirección de tareas y los equipos tecnológicos necesarios para realizarlas. Estas emociones bien podrían generar problemáticas más serias a nivel personal en los niños y adolescentes.

\section{Referencias Bibliográficas}

Anderete Schwal, M. (2020). Las desigualdades educativas durante la pandemia en la educación primaria de Argentina. CONICET Digital, 5-10.

Asociación Española contra el Cáncer. (12 de Agosto de 2021). aecc.es. https://www.aecc.es/sites/default/files/migration/actualidad/publicaciones/docu mentos/las-emociones.pdf

Barrientos Jiménez, E. (2013). Investigación Educativa. Ediciones Universidad Nacional Mayor de San Marcos. Lima, Perú.

Bueno y Torrens, D. (2019). Neurociencia para educadores. 4ta edición. Editorial Octaedro. Barcelona.

Del Moral, M., \& Villalustre, L. (2005). Adaptación de los entornos virtuales a los estilos cognitivos de los estudiantes: un factor de calidad en la docencia virtual. PixelBit. Revista de Medios y Educación, 16-25.

Duart, J. M., \& Sangrà, A. (2000). Aprender en la Virtualidad. Catalunya: Gediasa SA. https://d1wqtxts1xzle7.cloudfront.net/54876717/2000_DUART_Joseph_y_SAG RA_Albert-Formacion_Universitaria_por_medio_de_la_web- 
un_modelo_integrador-with-cover-page-

v2.pdf?Expires $=1625113541 \&$ Signature $=X 3 F T j V y S X 1 M b 8 d t P O c r F y Q N K F P N J$ 6FxgStMAYf4T7ur kxuizXmj3eVfJs

Fernández, R. (2017). Neuropedagogía: Hacia una educación cerebro- compatible. Editorial Bonu.

Gálvez, M. (2021). Pandemia y confinamiento: un desorden en los procesos del sueño. Rhombus. Universidad Latinoamericana de Ciencia y Tecnología , 50-61.

García, J. (2012). La educación emocional, su importancia en el proceso de aprendizaje. Educación, 1-25.

Garrido, J. (s.f.). psicopedia.org. https://psicopedia.org/1369/emociones-que-soncuantas-hay-como-determinan-nuestra-conducta/

Johnson, M., Saletti-Cuesta, L., \& Tumas, N. (2020). Emociones, preocupaciones y reflexiones frente a la pandemia del COVID-19 en Argentina. Ciênc. saúde coletiva, 2447-2456.

Lurich, F., Maurel, M. d., Aebicher, M., \& Mayol, Y. (2015). Perspectiva de los estudiantes en el uso de aulas virtuales como complemento de las clases presenciales. El caso del IESETyFP. X Congreso de Tecnología en Educación \& Educación en Tecnología (págs. 375-382). Corrientes: Instituto de educación Superior de Educación Técnico y profesional de la provincia del Chaco.

Ministerio de Educación del Ecuador. (2020). educacion.gob.ec. https://educacion.gob.ec/wp-content/uploads/downloads/2020/08/PlanEducativo-Aprendamos-Juntos-en-Casa.pdf

Ministerio de Educación del Ecuador. (11 de 08 de 2021). educacion.gob.ec. https://educacion.gob.ec/plan-educativo-aprendemos-juntos-en-casa/

Moll, S. (23 de enero de 2019). Educación 3.0. https://www.educaciontrespuntocero.com/noticias/tipos-de-aprendizajeselectronicos/

Sánchez, M., Martínez, A., Torres, R., de Agüero Servín, M., Hernández, A., Benavides, M., . . . Jaimes, C. (2020). Retos educativos durante la pandemia de COVID-19: una encuesta a profesores de la UNAM. Digital Universitaria, 1-24.

UNESCO, (2020), La educación en América Latina y el Caribe ante el COVID 19. https://es.unesco.org/fieldoffice/santiago/covid-19-education-alc

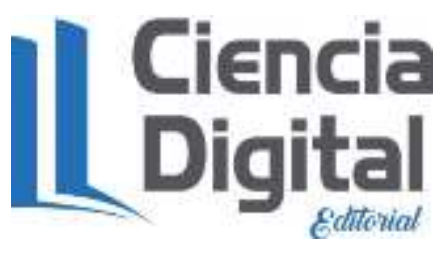




\section{PARA CITAR EL ARTÍCULO INDEXADO}

Flores Vélez, Ángela R., Cedeño Macías, L., Cedeño Zambrano, R., \& Vera Moreira, Ángela K. (2021). Las emociones de niños y adolescentes frente a la educación virtual debido a la crisis sanitaria . AlfaPublicaciones, 3(4.1), 85-95. https://doi.org/10.33262/ap.v3i4.1.126

\section{Ciencia}

El artículo que se publica es de exclusiva responsabilidad de los autores y no necesariamente reflejan el pensamiento de la Revista Alfa Publicaciones.

El artículo queda en propiedad de la revista y, por tanto, su publicación parcial y/o total en otro medio tiene que ser autorizado por el director de la Revista Alfa Publicaciones.
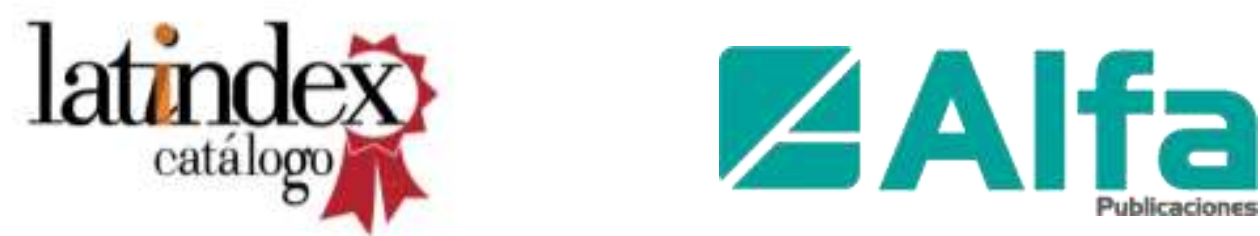